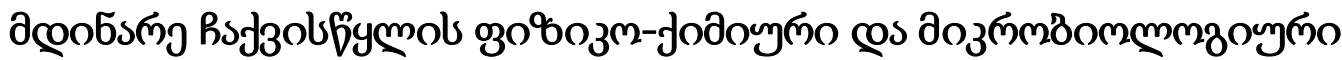

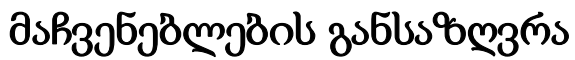

\author{
mmansosodg $606 m$

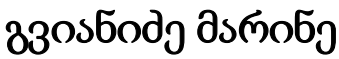

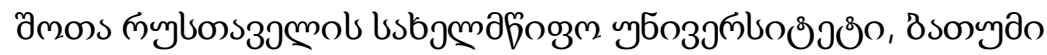

https://doi.org/10.52340/idw.2021.495

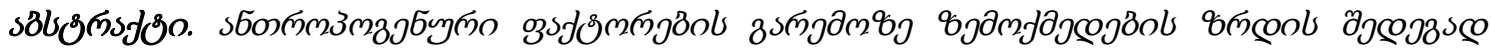

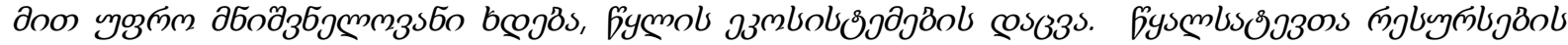

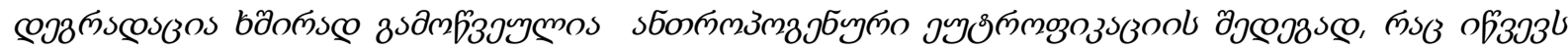

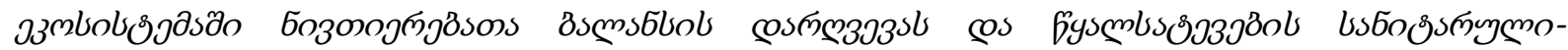

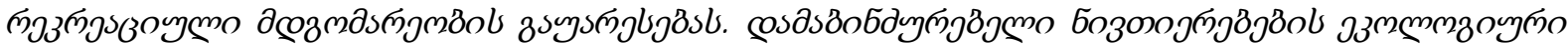

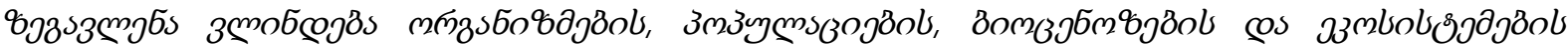

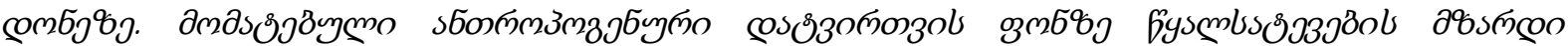

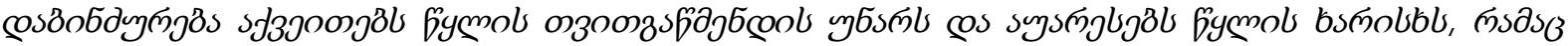

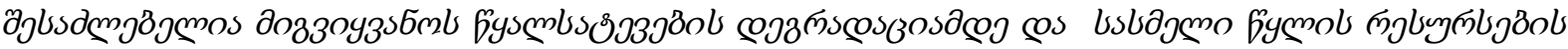

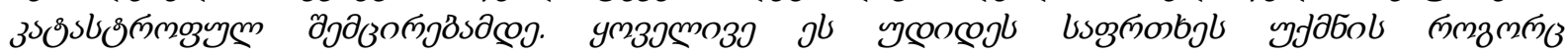

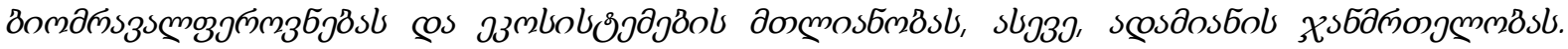

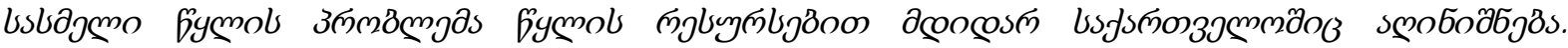

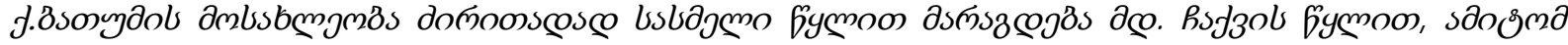

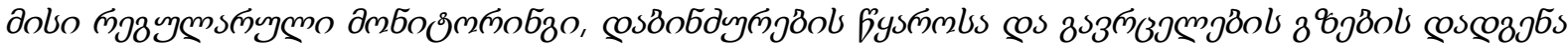

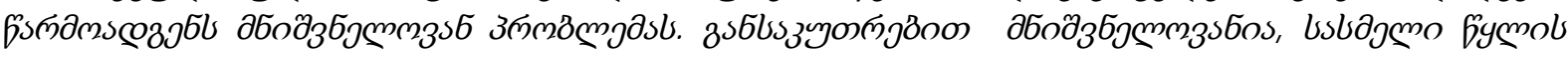

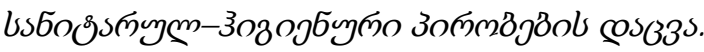

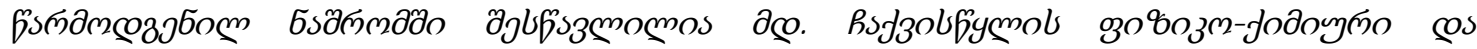

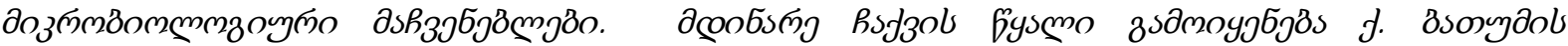

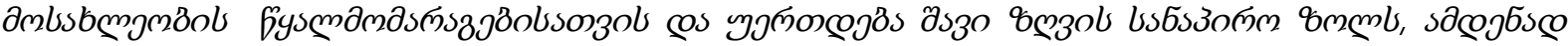

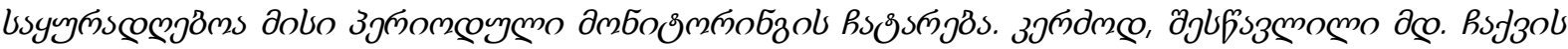

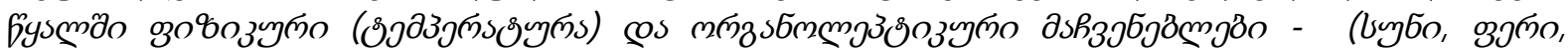

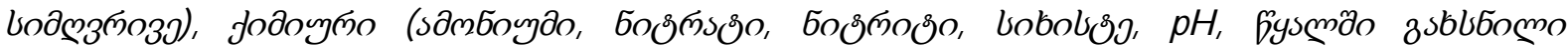

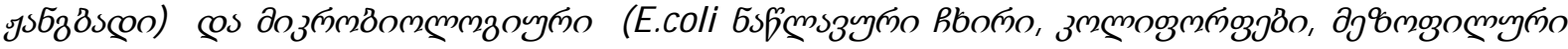

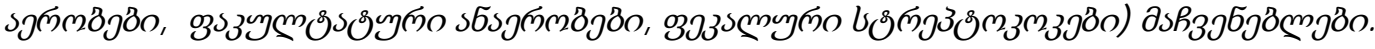

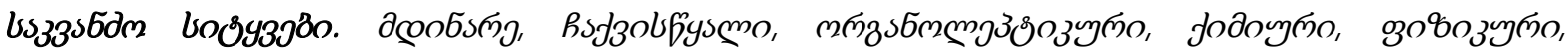

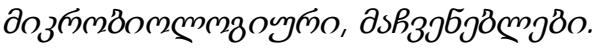

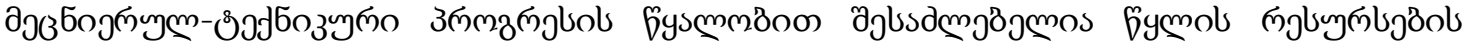

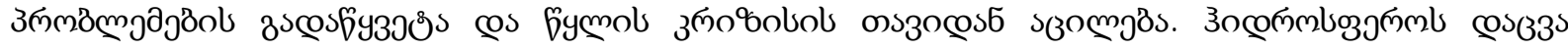

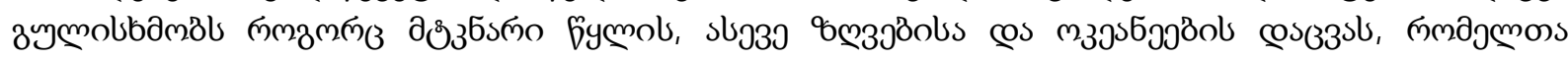

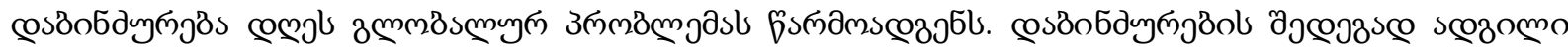

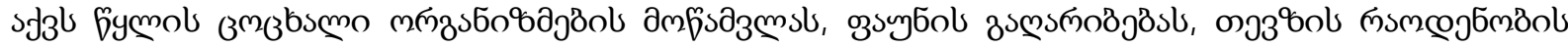

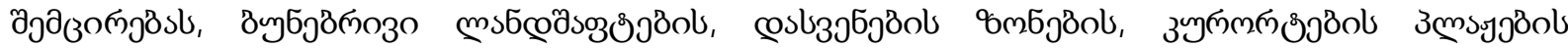

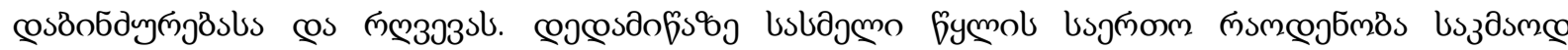

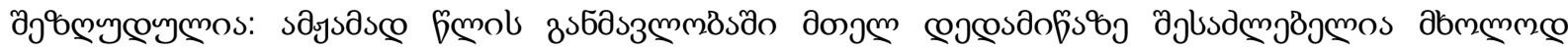

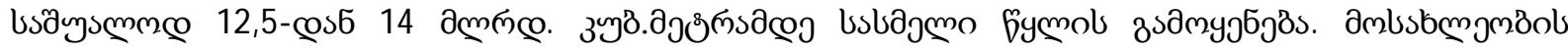




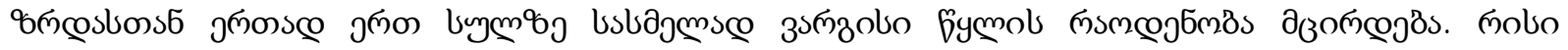

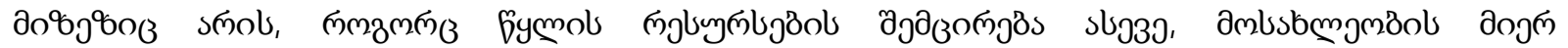

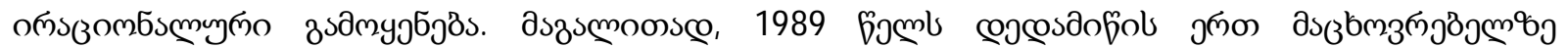

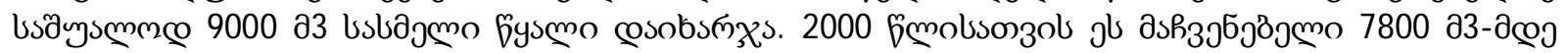

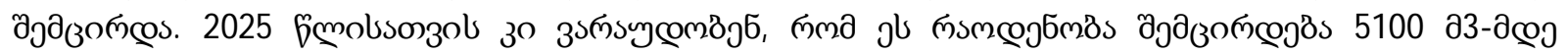

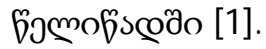

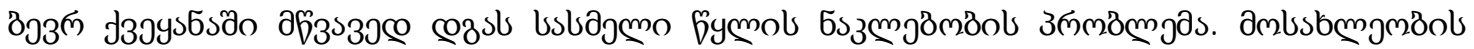

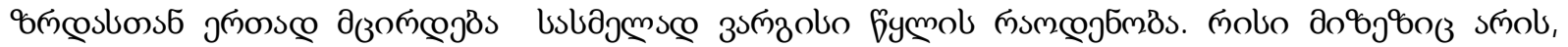

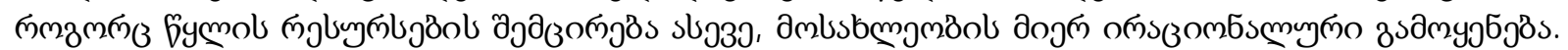

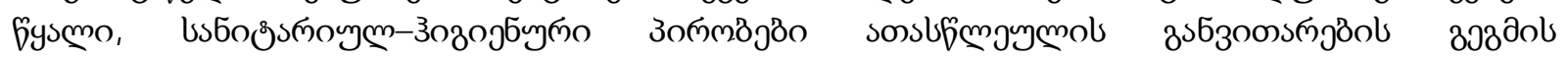

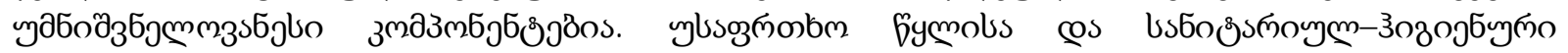

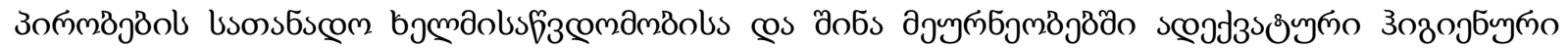

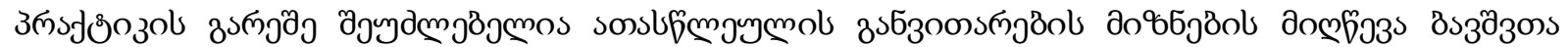

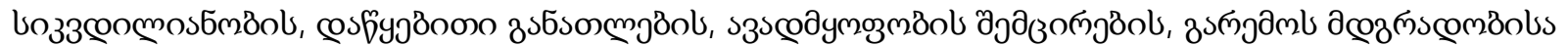

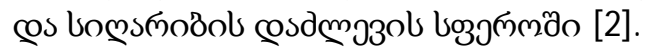

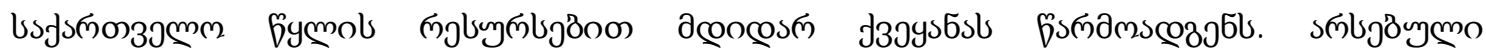

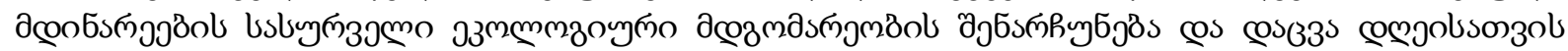

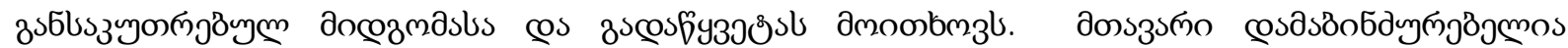

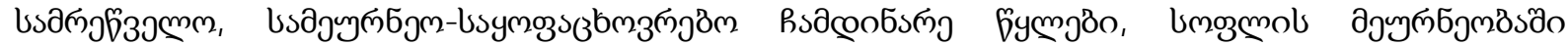

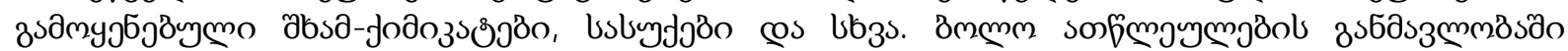

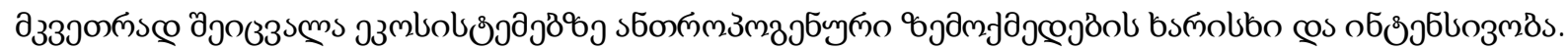

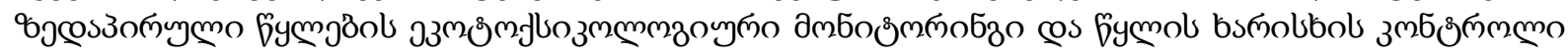

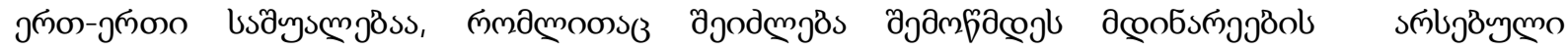

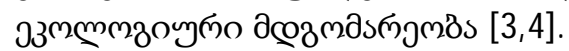

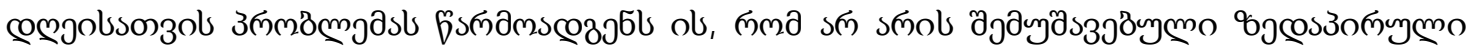

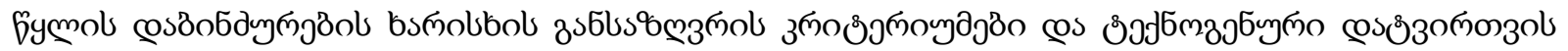

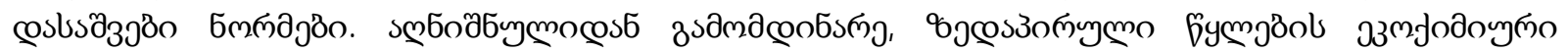

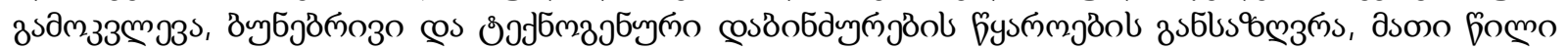

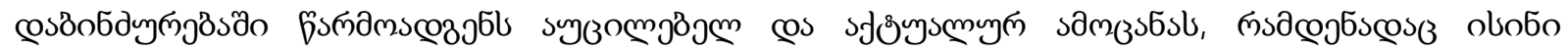

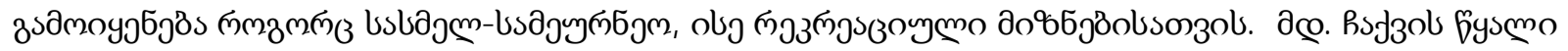

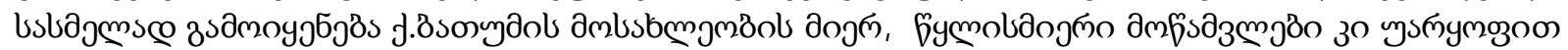

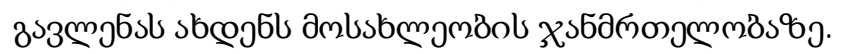

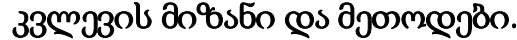

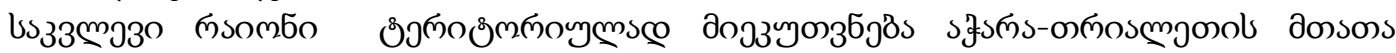

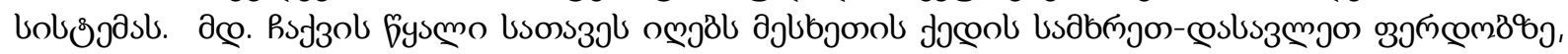

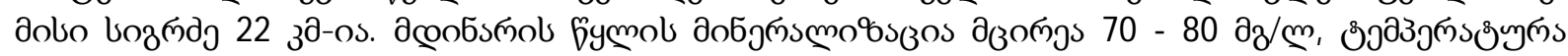

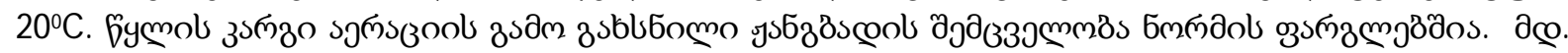

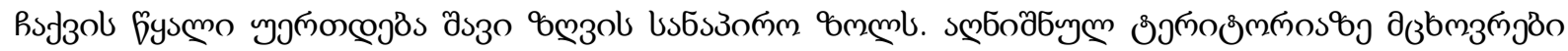

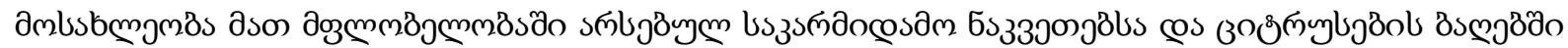

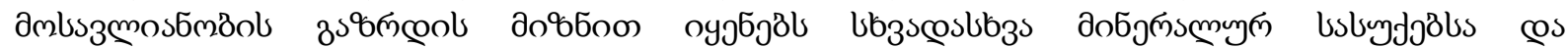

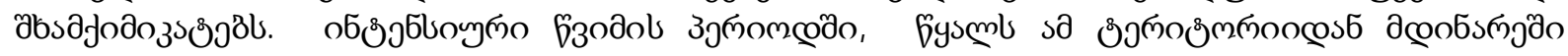

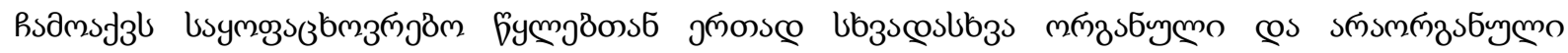

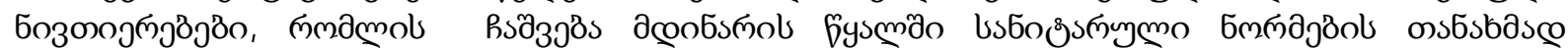

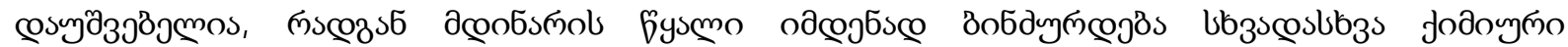

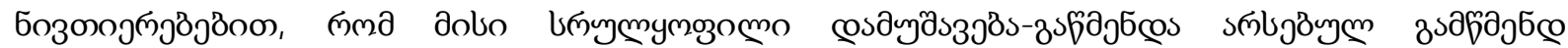

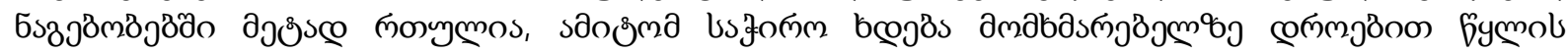

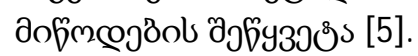

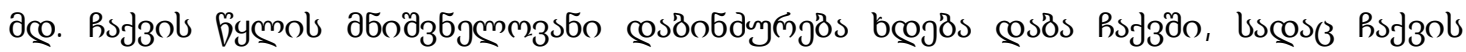




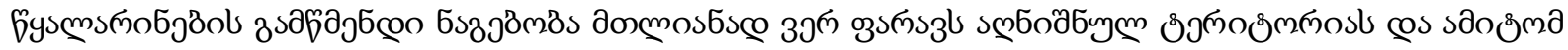

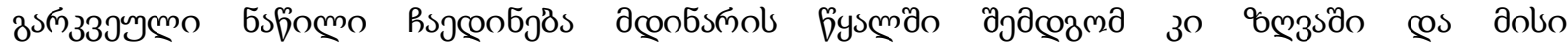

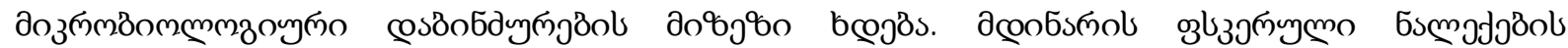

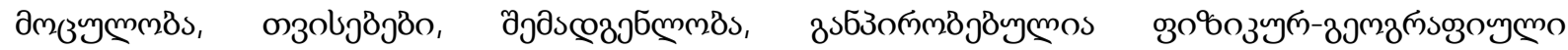

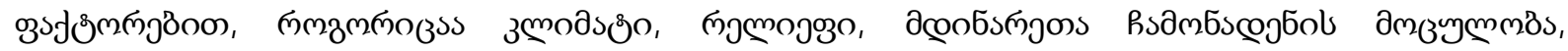

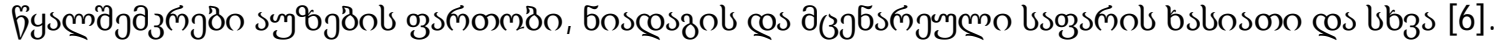

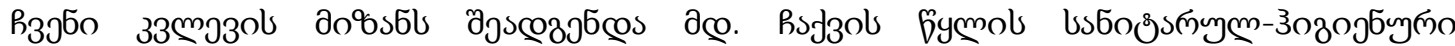

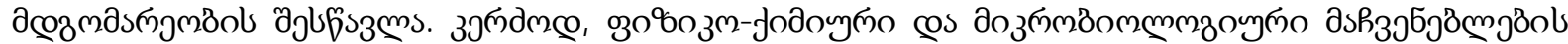

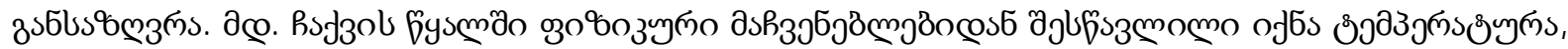

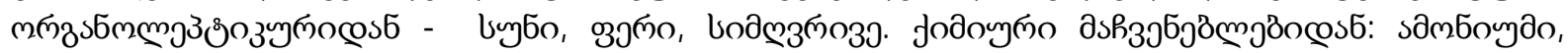

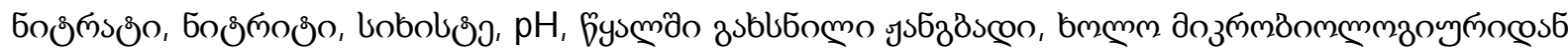

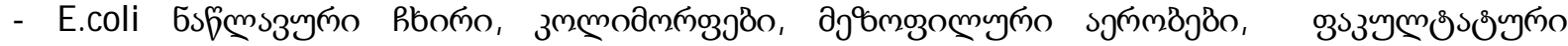

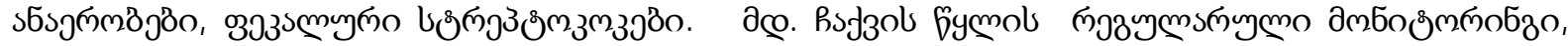

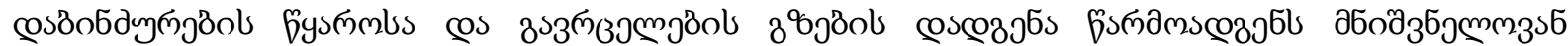

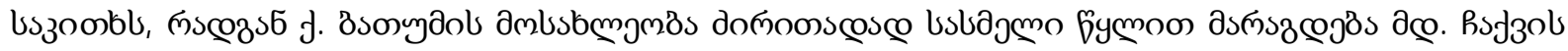
6ymogost.

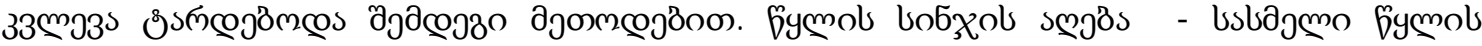

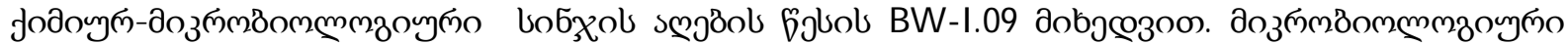

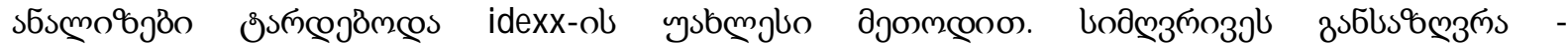

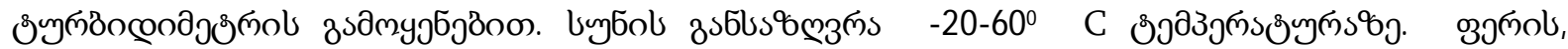

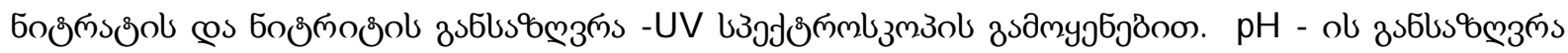

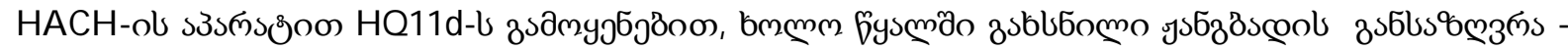

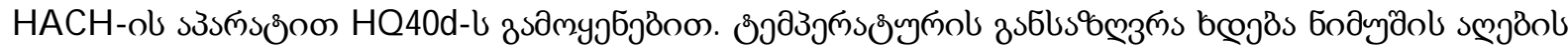

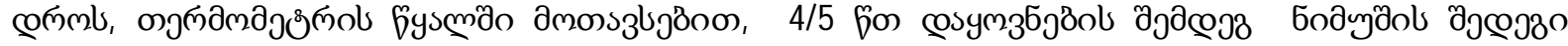

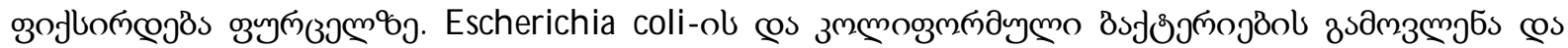

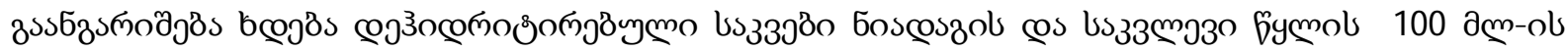

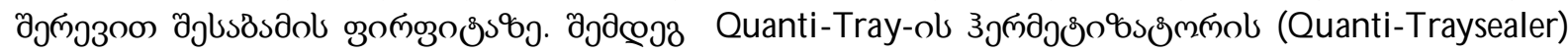

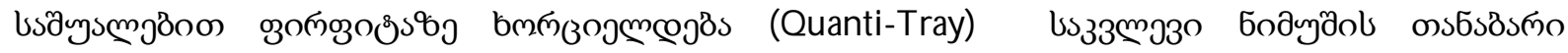

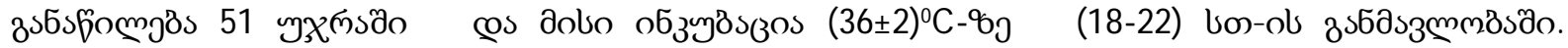

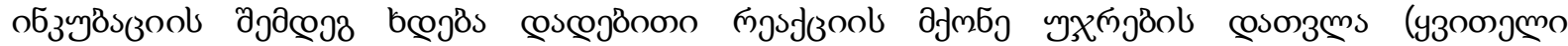

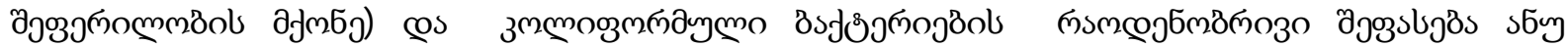

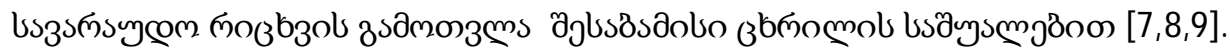

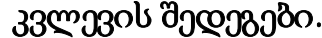

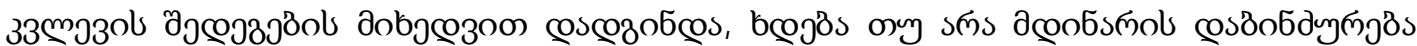

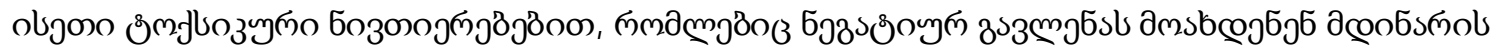

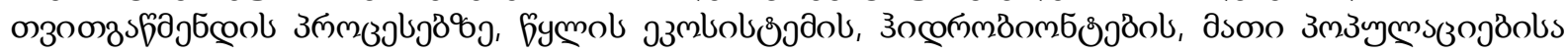

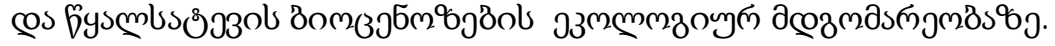

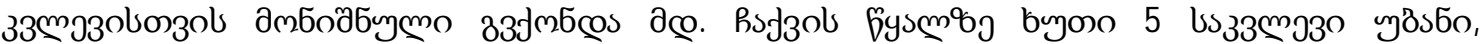

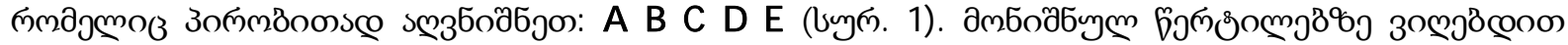

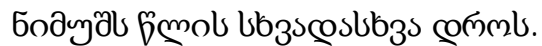




\begin{tabular}{|c|c|c|c|c|c|}
\hline \multicolumn{6}{|c|}{ 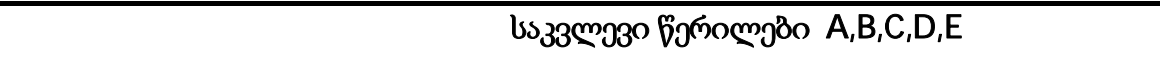 } \\
\hline зэตั & $0.0 \pm 0.0$ & $0.0 \pm 0.0$ & $\begin{array}{l}12.60 \pm 0,24 \\
P<0,0001\end{array}$ & $\begin{array}{l}4,800 \pm 0,200 \\
P<0,0001\end{array}$ & $60,00 \pm 0.0$ \\
\hline
\end{tabular}

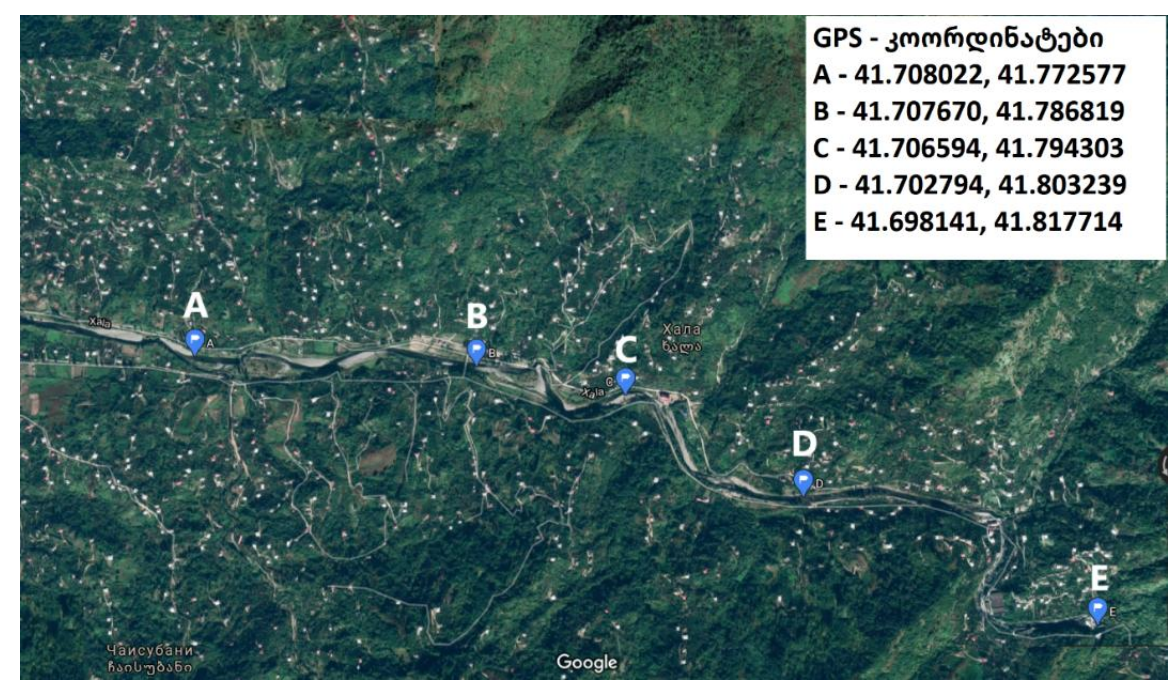

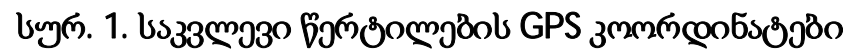

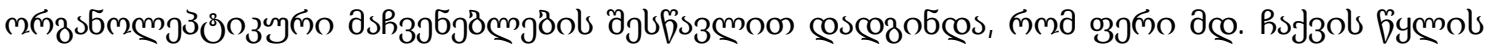

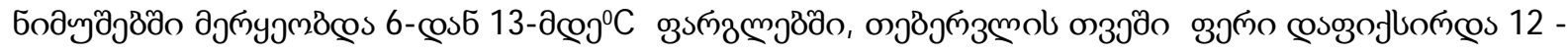

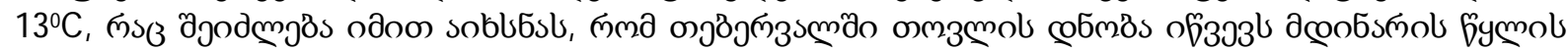

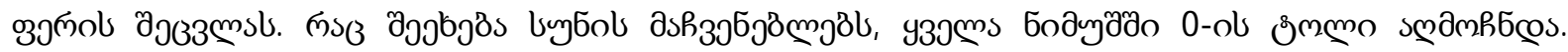

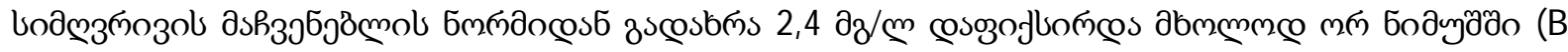

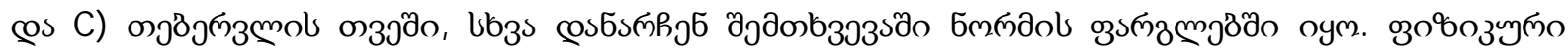

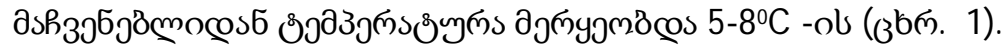

उbromo 1.

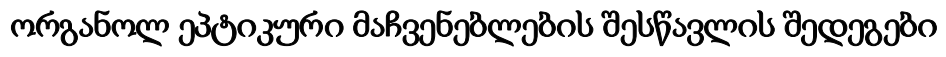




\begin{tabular}{|c|c|c|c|c|c|}
\hline \multicolumn{6}{|c|}{ 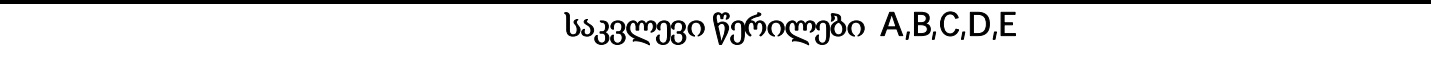 } \\
\hline 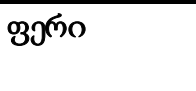 & $0.0 \pm 0.0$ & $0.0 \pm 0.0$ & $\begin{array}{l}12.60 \pm 0,24 \\
P<0,0001\end{array}$ & $\begin{array}{l}4,800 \pm 0,200 \\
P<0,0001\end{array}$ & $60,00 \pm 0.0$ \\
\hline byg6o & $0.0 \pm 0.0$ & $0.0 \pm 0.0$ & $0.0 \pm 0.0$ & $0.0 \pm 0.0$ & $0.0 \pm 0.0$ \\
\hline ঢодмзґоз & $\begin{array}{l}0,5680 \pm 0,0205 \\
P<0,0001\end{array}$ & $\begin{array}{l}1,680 \_ \pm 0,020 \\
P<0,0001\end{array}$ & $\begin{array}{l}2,340 \pm 0,024 \\
P<0,0001\end{array}$ & $\begin{array}{l}1,278 \pm 0,019 \\
P<0,0001\end{array}$ & $\begin{array}{l}1,168 \pm 0,0217 \\
P<0,0001\end{array}$ \\
\hline 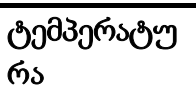 & $8.0 \pm 0.0$ & $\begin{array}{l}5,800 \pm 0,20 \\
p=0,001\end{array}$ & $7,0 \pm 0,0$ & $8.0 \pm 0.0$ & $\begin{array}{l}5,600 \pm 0,2449 \\
P<0,0001\end{array}$ \\
\hline
\end{tabular}

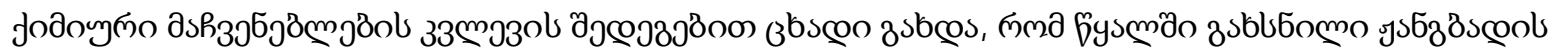

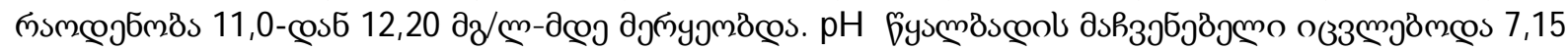

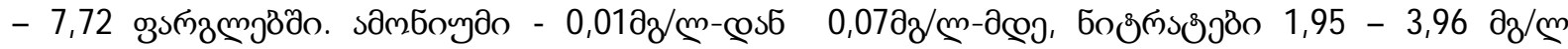

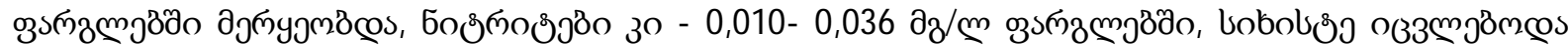

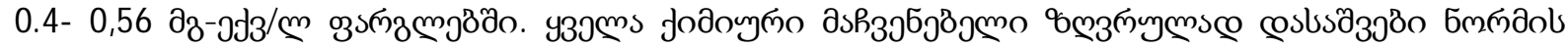

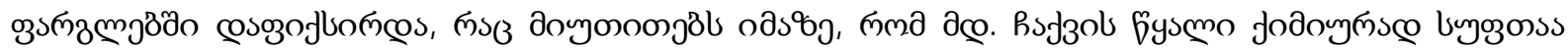
(зью. 2).

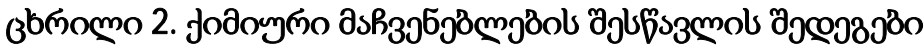

\begin{tabular}{|c|c|c|c|c|c|}
\hline \multicolumn{6}{|c|}{ 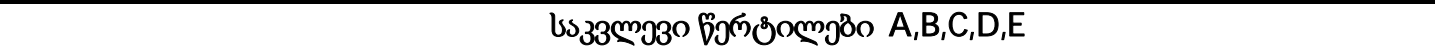 } \\
\hline 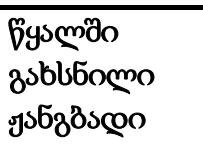 & $\begin{array}{l}11,37 \pm 0,22 \\
P<0,0001\end{array}$ & $\begin{array}{l}12.11 \pm 0,05 \\
P<0,0001\end{array}$ & $\begin{array}{l}11,98 \pm 0,08 \\
P<0,0001\end{array}$ & $\begin{array}{l}11,94 \pm 0,03 \\
P<0,0001\end{array}$ & $\begin{array}{l}11,39 \pm 0,21 \\
P<0,0001\end{array}$ \\
\hline 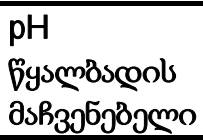 & $\begin{array}{l}7,686 \pm 0,001 \\
P<0,0001\end{array}$ & $\begin{array}{l}7,232 \pm 0,01 \\
P<0,0001\end{array}$ & $\begin{array}{l}7,326 \pm 0,0094 \\
P<0,0001\end{array}$ & $\begin{array}{l}7,18,0 \pm 0,00094 \\
P<0,00001\end{array}$ & $\begin{array}{l}7,337 \pm 0,01 \\
P<0,0001\end{array}$ \\
\hline samboyao & $\begin{array}{l}0,0140 \pm 0,002 \\
p=0,0046\end{array}$ & $\begin{array}{l}0,0280 \pm 0,0020 \\
p=0,0002\end{array}$ & $\begin{array}{l}0,260 \pm 0,0024 \\
p=0,0004\end{array}$ & $\begin{array}{l}0,0320 \pm 0,00020 \\
P<0,0001\end{array}$ & $0,007 \pm 0,0$ \\
\hline 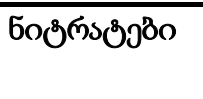 & $2,78 \pm 0,0$ & $\begin{array}{l}3,406- \pm 0,006 \\
P<0,0001\end{array}$ & $\begin{array}{l}3,548 \_ \pm 0,0037 \\
P<0,0001\end{array}$ & $\begin{array}{l}1,946 \pm 0,0067 \\
P<0,0001\end{array}$ & $\begin{array}{l}2,068 \pm 0,0276 \\
P<0,0001\end{array}$ \\
\hline 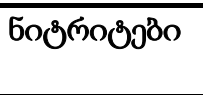 & $\begin{array}{l}0,0118 \pm 0,00037 \\
P<0,0001\end{array}$ & $\begin{array}{l}0,0348 \pm 0,00058 \\
P<0,0001\end{array}$ & $\begin{array}{l}0,010 \pm 0,00054 \\
P<0,0001\end{array}$ & $\begin{array}{l}0,0238 \pm 0,0003 \\
P<0,0001\end{array}$ & $\begin{array}{l}0,0238 \pm 0,0018 \\
P<0,0001\end{array}$ \\
\hline bobobతి? & $0,56 \pm 0,0$ & $0,56 \pm 0,0$ & $0,04 \pm 0,0$ & $0,04 \pm 0,0$ & $0,05 \pm 0,0$ \\
\hline
\end{tabular}

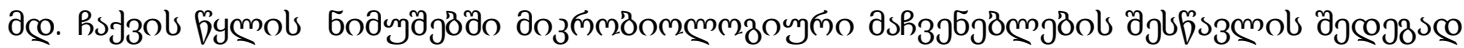

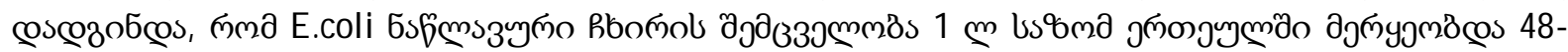

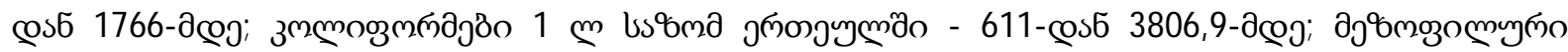

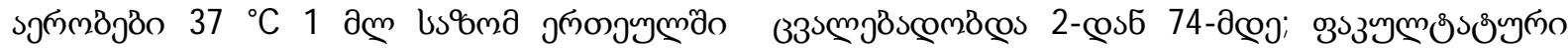

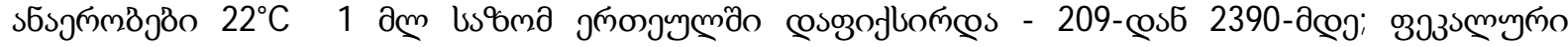

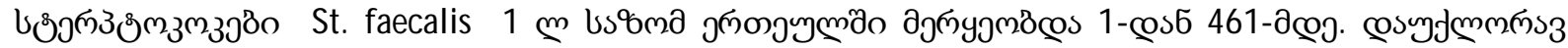

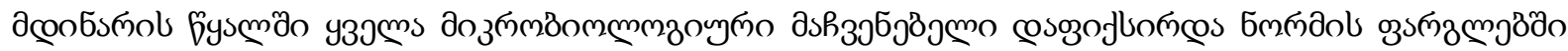
(зью. 3). 


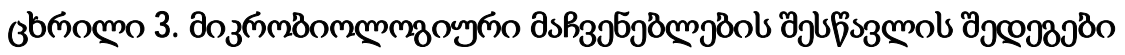

\begin{tabular}{|c|c|c|c|c|c|c|}
\hline \multicolumn{7}{|c|}{ Results of the study of microbiological indicators } \\
\hline 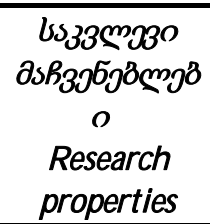 & $\begin{array}{l}\text { usqman } \\
\text { gromjymo } \\
\text { Units }\end{array}$ & 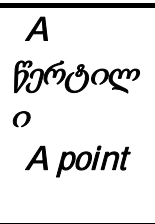 & $\begin{array}{l}B \\
\text { 6jூðீomo } \\
\text { B point }\end{array}$ & 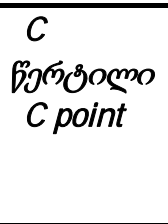 & 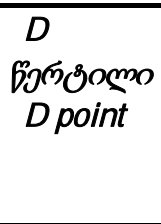 & 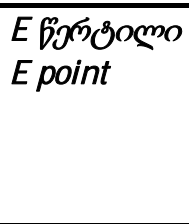 \\
\hline E.coli & 11 & 951,25 & 305 & 218 & 134 & 79 \\
\hline $\begin{array}{l}\text { coliform } \\
\text { bacteria }\end{array}$ & 11 & 3074 & 1466 & 1328 & 1428 & 641 \\
\hline $\begin{array}{c}\text { Aerobic } \\
\text { mesophilic } 37^{\circ} \mathrm{C}\end{array}$ & $1 \mathrm{ml}$ & 54,5 & 34 & 16 & 9 & 4 \\
\hline $\begin{array}{c}\text { Facultative } \\
\text { anaerobe } 22^{\circ} \mathrm{c}\end{array}$ & $1 \mathrm{ml}$ & 1254 & 784 & 623 & 680 & 475 \\
\hline $\begin{array}{l}\text { Streptococcus } \\
\text { faecal is }\end{array}$ & $1 \mathrm{ml}$ & 159 & 28 & 19 & 29 & 19 \\
\hline
\end{tabular}

\section{coslu33бj8:}

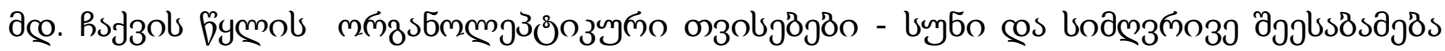

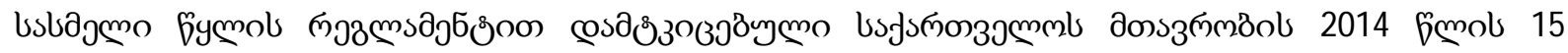

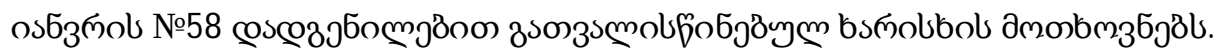

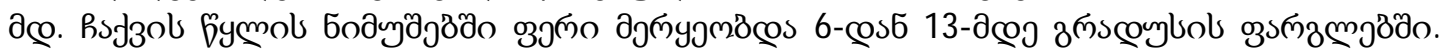

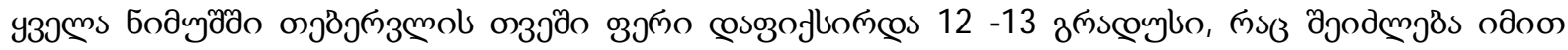

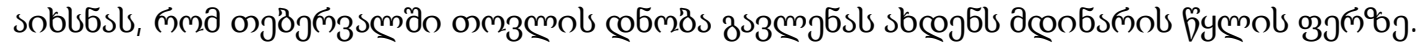

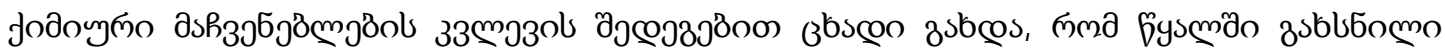

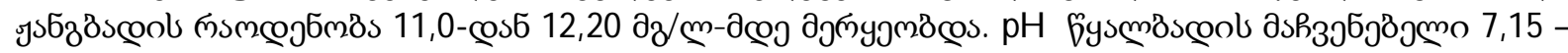

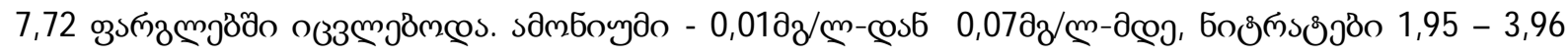

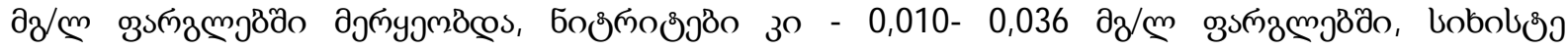

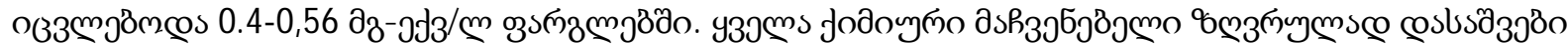

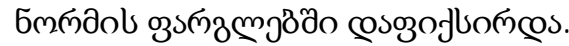

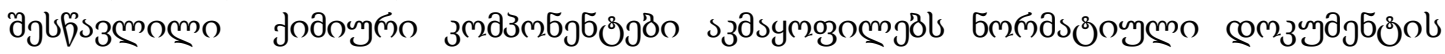

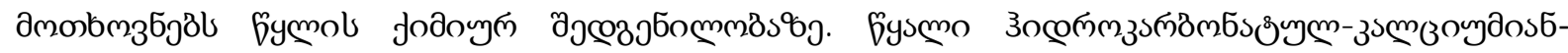

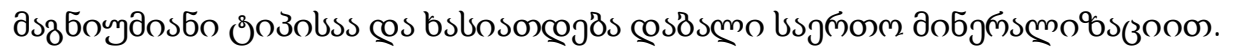

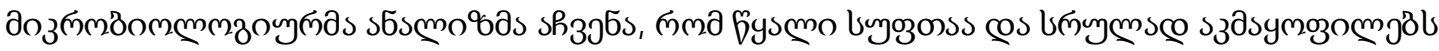

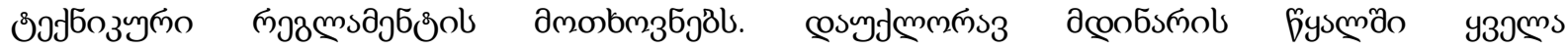

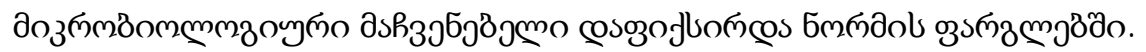

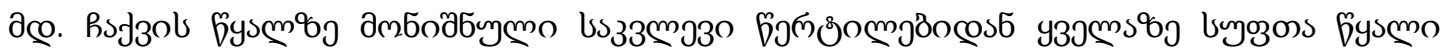

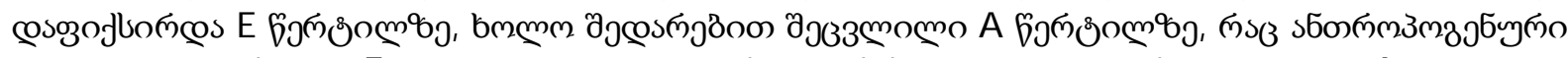

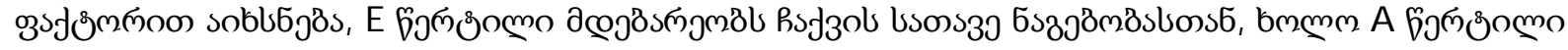

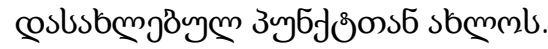

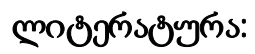

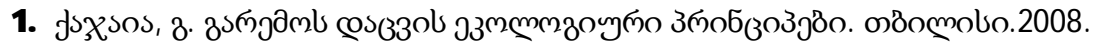

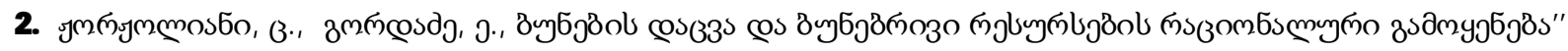
jyossobo. 2010

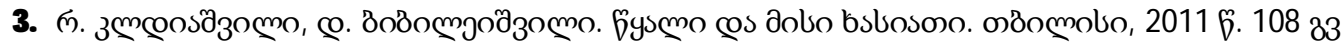




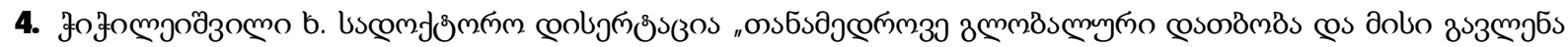

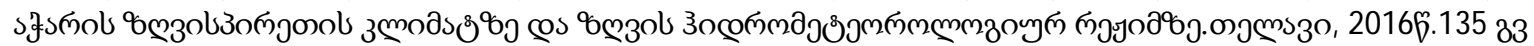

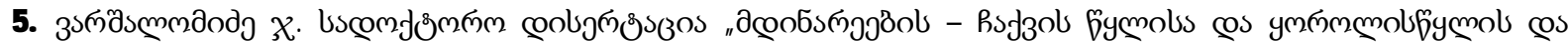

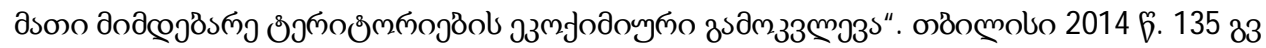

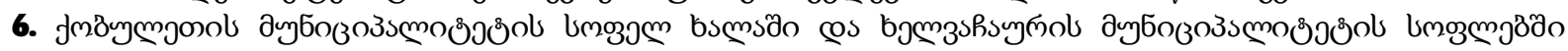

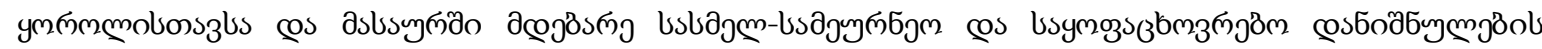

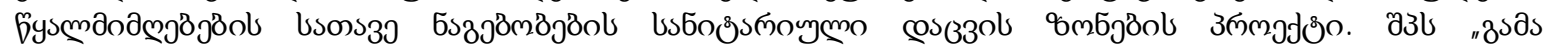

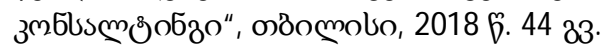

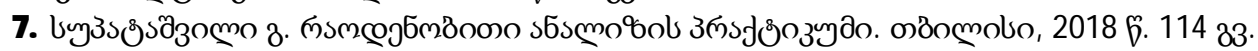

8. Золотова Ю. А. Основы аналитической химии. Практическое руководство под редакции М., изд. МГУ, 2001.

9. Харитонов Ю. Я. Количественный анализ. Изд. Высшая школа, М., 2010.

\title{
Determination of the Physicochemical and M icrobiological Index of the River Chakvistskali
}

\author{
Lomtatidze Nino \\ Gvianidze M arine
}

Shota Rustaveli State University, Batumi

\begin{abstract}
The growing impact of the anthropogenic factors on the environment has intensified the importance of protecting the water ecosystem. The degrading of water resources is a frequent result of the anthropogenic eutrophication resulting in imbalance of substance levels and deterioration of sanitation and recreational condition of water. The environmental impact of contaminants is manifested at the level of organisms, populations, biocenosis, and ecosystems. In the context of the increased anthropogenic load, the growing contamination of water bodies impairs the self-cleansing capacity of water, causes eutrophication and reduces water quality. Pollution of the natural water resources might lead to the degradation of water bodies and catastrophic reduction of the clean drinking water resources. This poses a huge danger to biodiversity and ecosystems in general, as well as human health. The problem of the drinking water exists in a country rich in water resources like Georgia as well. M ajority of population of Batumi receives drinking water from the river Chakhvistskali, therefore, its regular monitoring, and determining the sources of its pollution and ways of spreading represents and important issue.

The aim of our research is to study the physicochemical and microbiological properties of the water in the river Chakvi. The river water is used for supplying the city of Batumi with water flows into the Black Sea coast. The following objectives were set based on the research aim: to determine the physical (temperature) and organoleptic properties (odor, color, turbidity), chemical (ammonium, nitrates, nitrites, hardness, pH, dissolved oxygen), and microbiological (E.coli, coliform bacteria, aerobic mesophilic, facultative anaerobe, streptococcus faecalis) properties.
\end{abstract}

Key words: River, Chakvistskali, Physicochemical, Microbiological Index, properties 